\title{
Fault Diagnosis System Based on Multiagent Technique for Ship Power System
}

\author{
Zheng Wang, ${ }^{1,2}$ Li Xia, ${ }^{2}$ Yongji Wang, ${ }^{1}$ and Lei Liu' \\ ${ }^{1}$ School of Automation, Huazhong University of Science and Technology, Wuhan 430074, China \\ ${ }^{2}$ School of Electrical Engineering, Naval University of Engineering, Wuhan 430033, China \\ Correspondence should be addressed to Yongji Wang; wangyjch@mail.hust.edu.cn
}

Received 11 December 2013; Accepted 9 February 2014; Published 19 March 2014

Academic Editor: Huaicheng Yan

Copyright (c) 2014 Zheng Wang et al. This is an open access article distributed under the Creative Commons Attribution License, which permits unrestricted use, distribution, and reproduction in any medium, provided the original work is properly cited.

Fault diagnosis system of ship power system can assist the crew to deal with faults, shorten the processing time, and prevent faults expanding. Multiagent technique is adopted for the fault diagnosis system. Ship power system is divided into several feeder units. Each one is abstracted as a regional feeder agent (FED-Agent). A multiagent fault diagnosis system is established with FEDAgent and other functional agents. Considering of the characteristics of agent, the multiagent system processes both autonomy and interactivity. It can solve fault diagnosis problem of ship power system effectively.

\section{Introduction}

Modern ship power systems become larger and more complex, and once faults occur, there will be a great deal of alarm information. Although most ship power monitoring systems at present have some simple fault diagnosis functions, but when some failures occur, they just provide numerous pieces of alarm information to the crew unselectively. That is very difficult for the crew to determine fault quickly and accurately. So the research on fault diagnosis techniques for ship power system is an interesting topic. It can assist the crew to deal with power system faults, shorten the processing time, and prevent faults expanding. Fault diagnosis system plays an important role for ensuring safe and stable operation of ship power systems [1-3].

Currently, researchers have proposed many kinds of fault diagnosis methods, such as diagnosis method based on expert system, neural network, and information fusion. Simulation results show methods that can effectively improve the rapidity of ship power system fault diagnosis [4-15]. However, by the above methods, diagnosis information is required to converge to the centralized control center for analysis and processing, which requires that all pieces of information must be totally correct. Once there is incomplete information, false information, or conflicting information, the accuracy of fault diagnosis is hard to be guaranteed. This is the defect of "centralized" fault diagnosis techniques. To solve this problem, multiagent techniques can be used.

\section{Multiagent System and Its Application in Ship Power System}

2.1. Simplified Network Model of Ship Power System. Large ship power system is usually made up of several power stations; every station is composed of several generators. A ring or ladder power system is built by loop switches among buses and main switchboards, and the loads get power from buses or distribution switchboards [16].

The research of this paper is based on the typical threepower-station ladder shipboard power system [17]; its simplified network model is given in Figure 1.

The system is made up of 6 generators G, 18 loads (including static loads I and motors M), 10 feeders F, and 6 jumper wires $\mathrm{L}$. The switchboards and jumper wires divide the whole system into three power stations. The alternative routes of important loads are connected by dashed lines. 


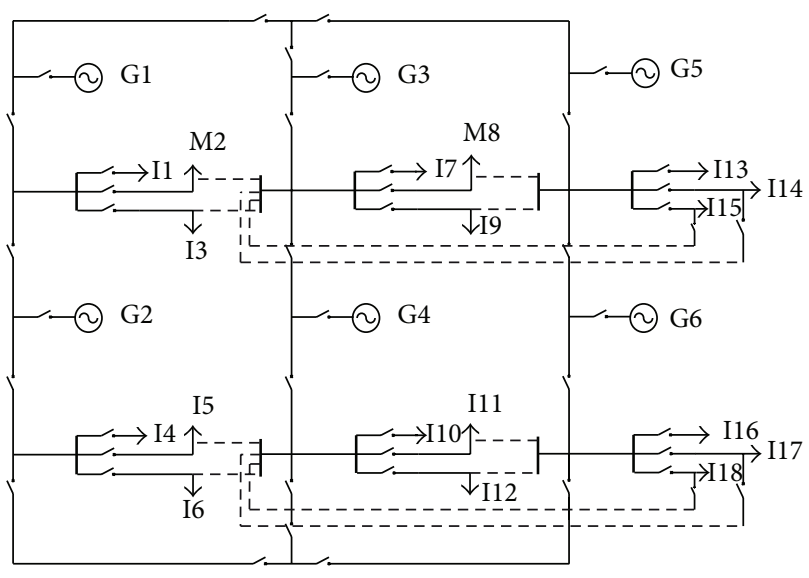

FIGURE 1: Simplified network model of SPS.

According to the graph theory, in Figure 1, the electrical devices and wires (including generators, loads, jumper wires, and switches) are abstracted to be branches, while the connection points between the devices are to be nodes, and then get a node-branch topology, as shown in Figure 2. There are totally 15 nodes and 51 branches in the whole system.

In Figure 2, nodes and branches get their number according to depth-first search and breadth-first search of graph theory; that is, $0 \sim 51$ in the circle represents branches and $0 \sim 15$ in the square represents nodes.

2.2. Multiagent System for Fault Diagnosis. Agent and Multiagent System (MAS) originate in the field of artificial intelligence. Because of its advantages, MAS is now one of main research domains of distributed artificial intelligence. Generally, agent is an entity with active behavioral capacity in any environment, such as organism, software system, or controller in control system. MAS is a loose coupling network formed by several agents. Physically or logically the agents are scattered, and their behaviors are self-governing. That is to say, their targets and behaviors would not be restricted by any other agents. To achieve the same task or the same goal, all agents link with each other under some kind of protocol. They can solve problems beyond single agent's capability by communication and cooperation [18-20].

Considering of distributed characteristics of multiagent, the set of nonswitch devices controlled by switch devices on the same regional feeder can be regarded as one agent. Therefore, the ship power system can be divided into several feeder units. Each one is abstracted as a regional feeder agent (FED-Agent). A regional feeder multiagent network model is proposed, as shown in Figure 3.

In its precinct, a FED-Agent can collect and analyze relevant information for fault diagnosis, so as to find faults in real time accurately, and forward fault information to other agents and upper centralized control unit. By communication and cooperation with each other, MAS can implement distributed system-level fault diagnosis.

\section{Research on Multiagent Fault Diagnosis System}

3.1. Structure of Multiagent Fault Diagnosis System. According to the characteristics of ship power system, a distributed multiagent fault diagnosis system is established in Figure 4.

In this system, there are distributed parallel architecture FED-Agent (regional feeder agent) at the bottom, SCM-Agent (subcommunication manage agent) in the middle layer, and FDI-Agent (fault diagnosis and infusion agent), PDB-Agent (primary data base agent), BDB-Agent (backup data base agent), and OPC-Agent (OPC communication agent) in the upper layer.

FED-Agent implements fault diagnosis in its precinct by fuzzy cognitive map reasoning and fuzzy relational contract net collaboration. The information of fault diagnosis will communicate with other FED-Agent and upload to the FDIAgent by way of SCM-Agent. By means of communication and cooperation of multiagent, fault diagnosis of ship power system is implemented.

3.2. Internal Logical Structure of Agent. The internal logical structure of FED-Agent is BDI (Belief-Desire-Intention) framework logical structure model based on decision making [21].

BDI framework structure is an important representative of the deliberative type agent. It imitates the process of a series of actions to achieve certain goals that people have taken. It is composed of three basic concepts, which are belief, desire, and intention. Belief reflects the cognitive characteristics of agent, desire reflects the preference feelings of agent, and intention reflects the goal of agent.

Define the agent structure based on BDI framework as follows:

$$
\text { Agent }=\{A, E, B, D, I, A \text {, see, bmp, opt, filter, exe }\}
$$

In which, $A$ represents the set of agents. $E$ represents the basic set of event types. Define Bel as a set of agent's all possible beliefs, so $B \in \gamma(\mathrm{Bel})$ represents one certain set of beliefs; define Des as a set of agent's all possible desires, so $D \in \gamma$ (Des) represents one certain set of desires; define Int as a set of agent's all possible intentions, so $I \in \gamma$ (Int) represents one certain set of intentions. According to the definitions above, the internal behavior of agent can be represented as follows:

see: $S \longrightarrow P$ represents the process of belief determining;

bmp: $\gamma(\mathrm{Bel}) \times P \longrightarrow \gamma(\mathrm{Bel})$ represents the process of

belief revision;

opt $: r(\mathrm{Bel}) \times r(\mathrm{Int}) \longrightarrow r(\mathrm{Des})$ represents the process

of desire determining; 


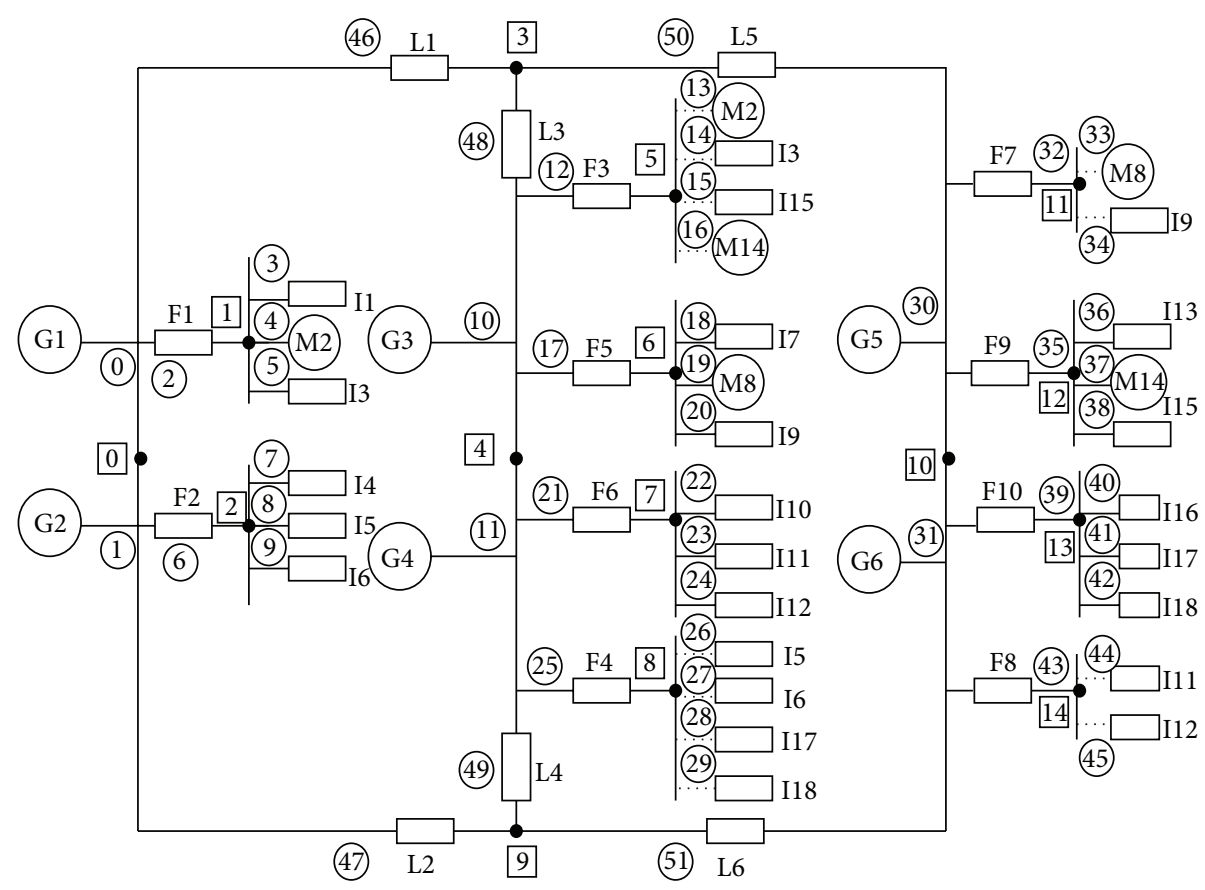

FIGURE 2: Ladder ship power system.

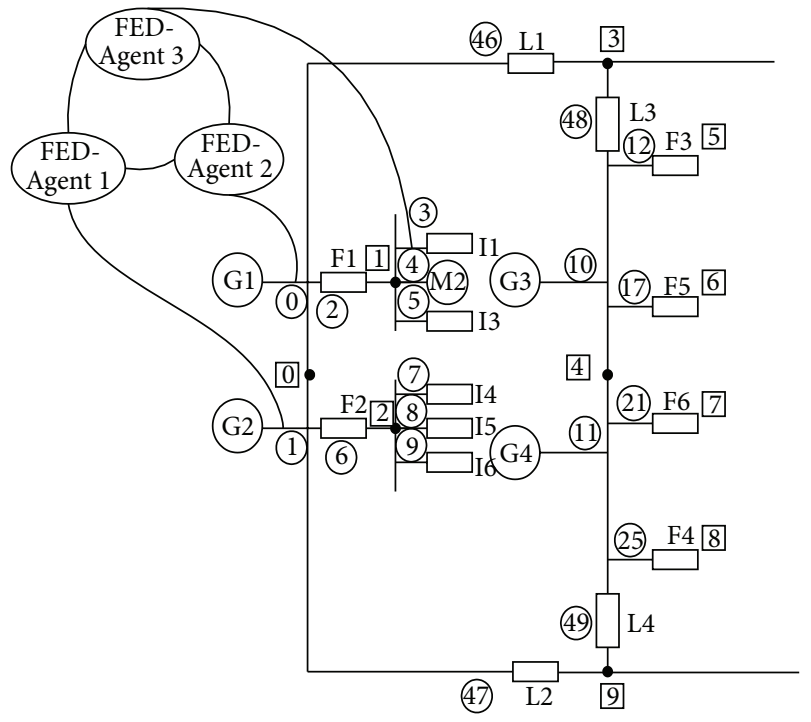

FIGURE 3: Regional feeder multiagent network model of ship power system.

filter: $\gamma($ Bel $) \times \gamma($ Des $) \times \gamma($ Int $) \longrightarrow \gamma($ Int $)$ represents the process of intention determining;

exe $\gamma($ Int $) \longrightarrow A$ represents specific behavior for a certain intention.

The agent structure based on BDI framework is shown in Figure 5.
Since the BDI framework is based on the theory of practical reasoning, it has a profound basis of cognitive psychology and philosophy, so agent's structure established is conformed to the trend of artificial intelligence. Meanwhile, it is difficult to express the concepts of belief, desire, and intention and complete the appropriate reasoning conversion in the actual structure of agent. To establish mature agent model based on BDI framework, an accurate description of the appropriate methods for agent knowledge reasoning process and communication with the environment is needed.

3.3. Main Functions of Multiagent Fault Diagnosis System. The aim of multiagent fault diagnosis system is to find out the faults in ship power system in real time and isolate and exclude the faults. To guarantee stable operation of SPS, the following functions of the fault diagnosis system are needed:

(1) monitoring and fault detection: each FED-Agent collects fault diagnosis information in its precinct and communicates with other FED-Agents to diagnose regional faults collaboratively. By cooperating with SCM-Agent and FDI-Agent, system-level faults can be diagnosed.

(2) Fault notification in real time (warning): simple fault information is delivered to SCM-Agent in real time, and complex fault diagnosis is implemented by FDIAgent. Alarms are displayed immediately on the GUI to the maintenance staff to ensure that fault is processed timely.

(3) Alarm status settings and active alarm list: setting alarm status of the corresponding device after alarm and maintaining active alarm list for finding out 


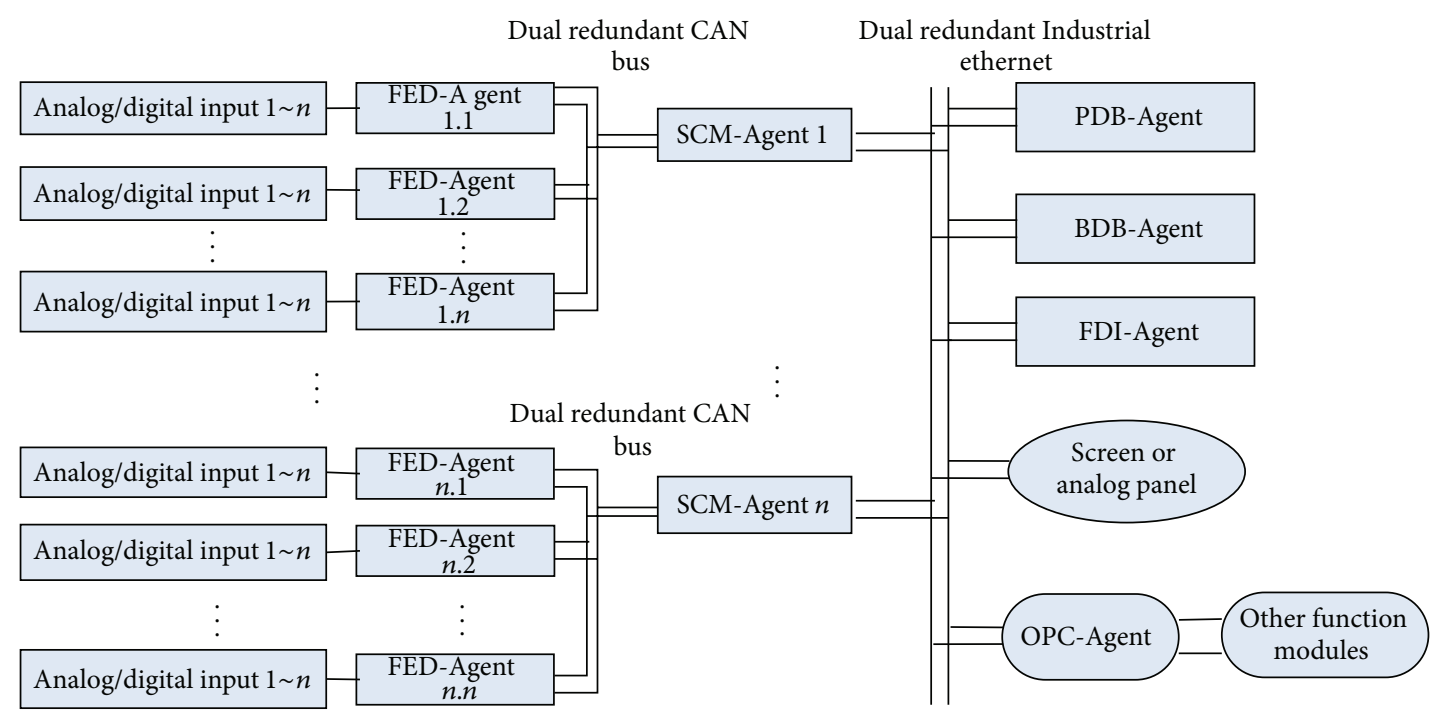

FIGURE 4: Multiagent fault diagnosis system for ship power system.

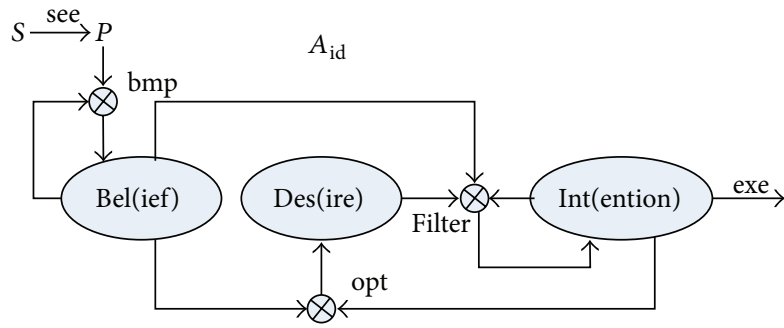

FIGURE 5: Agent structure based on BDI framework.

the current alarm status and detailed active alarm information.

(4) Alarm clear: clearing the corresponding alarm status and active alarm information of the fault device after excluding faults; automatic clearing and artificial clearing are both supported.

(5) Alarm filtration: setting alarm filtering conditions for selective alarm.

(6) Fault isolation: stopping the faulty pieces of equipment.

(7) Failure analysis: analyzing the causes of faults and restoring the device to work properly.

(8) Alarm history data saving: collecting and saving the alarm information for future alarm analysis.

3.4. Management and Cooperation of Multiagent. To ensure effective cooperation among multiagents, fuzzy contract net model is adopted. In this model, agents can take advantage of master-slave relationship with each other, so that information resources can be fully utilized to implement fault diagnosis [22-25].

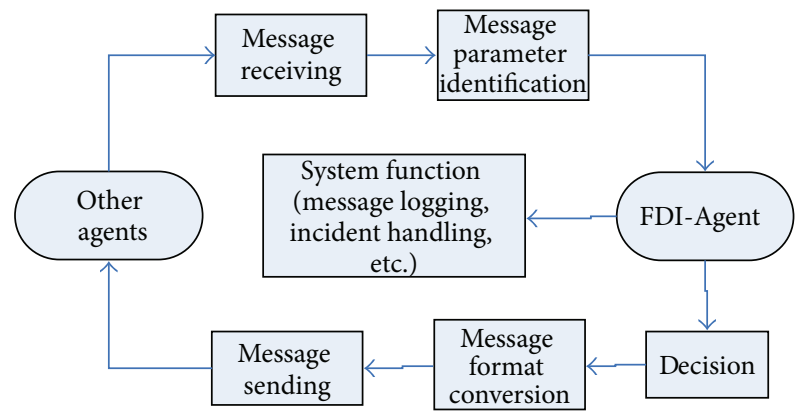

FIGURE 6: Message communication schematic diagram of multiagent fault diagnosis system.

Message communication mode is adopted among FEDAgents and between FED-Agent and SCM-Agent. This communication mode is also adopted between SCM-Agent and FDI-Agent. Message communication is simple; three conditions are needed to meet: first, there should be a communication protocol. Second, common communication language among multiagents is needed. Third, common understanding for the language is required.

From the perspective of system implementation, agent is just like object; the interface independent of its internal data structure and message-based is needed to be provided. In the object-oriented field, communication is called by method; the meaning of message is different for each object. But in the agent-oriented software engineering, common language is used by message, regardless of agent's semantics.

The message communication schematic diagram of multiagent fault diagnosis system is shown in Figure 6.

Because of message communication adopted, there are some characteristics of communication in multiagent fault diagnosis system as following: communication data is less, which will shorten network transmission time, reduce time delay, and improve real time of the system. The collaborative 
behavior described in message belongs to senior semantic information, so that there are low degrees of coupling and high heterogeneity among cooperative application programs. That is beneficial to build distributed and heterogeneous system. A hierarchical communication structure is designed, the communication protocol specification is presented, and the appropriate communication language and the messagebased communication mode are adopted in the paper. So the agents of multiagent fault diagnosis system can communicate and cooperate effectively, and implement distributed fault diagnosis.

3.5. Database Agent. Database agents provide data support for detailed design, programming, and testing of multiagent fault diagnosis system. There are PDB-Agent and BDB-Agent, and both of them have almost the same structure.

We select Oracle Company's database product, because it has perfect functions and high reliability and is easy to operate and maintain easy access to technical support. SCM-Agent is implemented by calling OCI (Oracle Call Interface), which is provided by ORACAL database. FDIAgent has access to ODBC (Open Database Connectivity) by calling ADO (ActiveX Data Objects), which is provided by Microsoft, so as to access to ORACAL database.

\section{Algorithm and Simulation}

4.1. Aim Function of Multiagent Fault Diagnosis System. There are many problems in fault diagnosis models in existence. For example, the fault diagnosis model shown as Formula (3) has multiple solutions [26]. We analyze the cause of multiple solutions; the improved fault diagnosis model considering the influence of each protection and the joint influence between the main and back-up protections is presented, shown as Formula (4)

$$
\begin{aligned}
E(X)= & \sum\left|r_{k m}-r_{k m}^{*}\right|+\sum\left|r_{k p}-r_{k p}^{*}\right| \\
& +\sum\left|r_{k s}-r_{k s}^{*}\right|+\sum\left|C_{i}-C_{i}^{*}\right|, \\
E(X)= & \sum\left|r_{k m}-r_{k m}^{*}\right|\left|1-r_{k p} r_{k p}^{*}-\stackrel{\oplus}{\sum} r_{k s} r_{k s}^{*}\right| \\
& +\sum\left|r_{k p}-r_{k p}^{*}\right|\left|1-\sum r_{k s} r_{k s}^{*}\right| \\
& +\sum\left|r_{k s}-r_{k s}^{*}\right|+\sum\left|r_{\mathrm{jmal}}-r_{\mathrm{jmal}}^{*}\right| \\
& +\sum\left|C_{i}-C_{i}^{*}\right|\left|1-r_{\mathrm{imal}} r_{\mathrm{imal}}^{*}\right| .
\end{aligned}
$$

In the Formulas (3) and (4), the continuous-or-operation is represented to be $\stackrel{\oplus}{\Sigma}$, the main protection of certain element real and anticipant estate is represented to be $r_{k m}$ and $r_{k m}^{*}$, the near backup protection of certain element real and anticipant estate is represented to be $r_{k p}$ and $r_{k p}^{*}$, the remote backup protection of certain element real and anticipant estate is represented to be $r_{k s}$ and $r_{k s}^{*}$, the breaker's real and anticipant estate is represented to be $C_{i}$ and $C_{i}^{*}$, and breaker failure

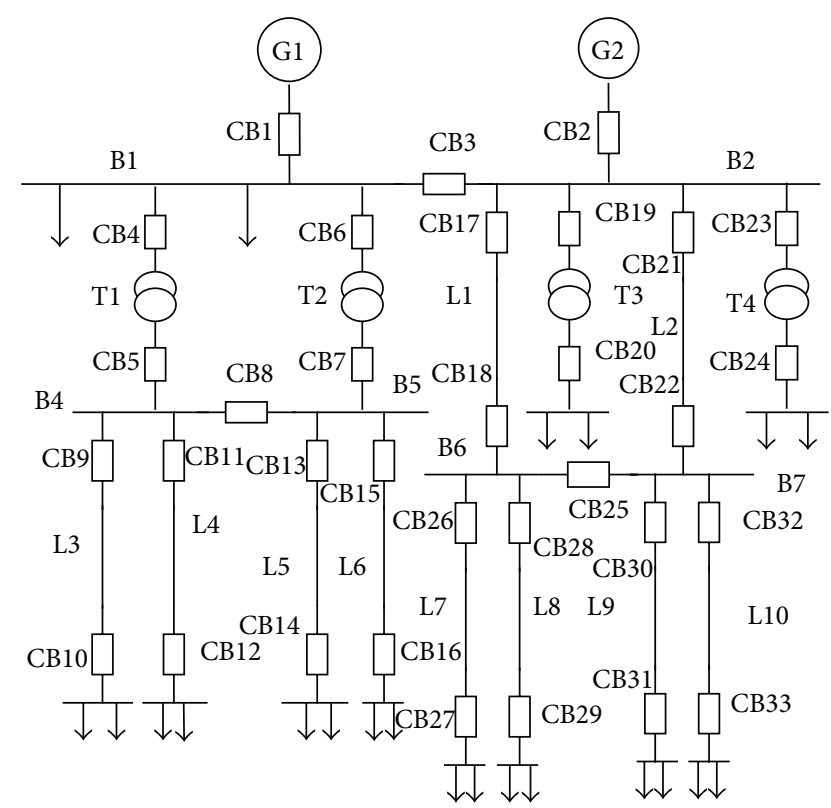

FIgURE 7: Power station 1st of ship power system.

protection's real and anticipant estate is represented to be $r_{\text {jmal }}$ and $r_{\text {imal }}{ }^{*}$.

In ship power system, the elements which are linked with the load directly only have the main protection and near backup protection. The remote backup protection is only provided by the element which is near to the source. So we can get the aim function of multiagent fault diagnosis system for ship power system as Formula (5)

$$
\begin{aligned}
E(X)= & \sum\left|r_{k m}-r_{k m}^{*}\right|\left|1-r_{k p} r_{k p}^{*}-r_{k s} r_{k s}^{*}\right| \\
& +\sum\left|r_{k p}-r_{k p}^{*}\right|\left|1-r_{k s} r_{k s}^{*}\right| \\
& +\sum\left|r_{k s}-r_{k s}^{*}\right|+\sum\left|C_{i}-C_{i}^{*}\right| .
\end{aligned}
$$

4.2. Example. Power station 1st of ship power system in Figure 2 is taken as example object, shown in Figure 7.

This system has 20 elements, 33 breakers, and 50 protections. $\left(S_{1} \sim S_{20}\right)$ are represented by 20 elements: B1,..., B6; $\mathrm{T} 1, \ldots, \mathrm{T} 4 ; \mathrm{L} 1, \ldots, \mathrm{L} 10 ;\left(C_{1} \sim C_{33}\right)$ are represented by 33 breakers: CB1, CB2,..., CB33; $\left(r_{1} \sim r_{20}\right)$ are represented by 20 main protections: B1m, ..., B6m; T1m, ..., T4m; L1m, ..., L10m; $\left(r_{21} \sim r_{40}\right)$ are represented by 20 near backup protections: B1p, .. , B6p; T1p, ... , T4p; L1p, .. , L10p; $\left(r_{41} \sim r_{50}\right)$ are represented by 10 remote backup protections: $\mathrm{B} 1 \mathrm{~s}, \ldots, \mathrm{B} 6 \mathrm{~s}$; T1s, T2s; L1s, L2s.

If there are faults that occur in the system, we get the warning signal: the protections of T1p, B1s, T2 m, and L5p and the breakers of CB5, CB3, CB1, CB6, CB7, and CB13 act. The elements in the power cut area are B1, B3, B4, T1, T2, L3, L4, L5, and L6 by the network topology analysis. 
TABle 1: Protections anticipant estates.

\begin{tabular}{|c|c|c|}
\hline $\begin{array}{l}r_{1}^{*}=s_{1} \\
r_{4}^{*}=s_{2} \\
r_{7}^{*}=s_{3} \\
r_{10}^{*}=s_{4} \\
{r_{13}}^{*}=s_{5}\end{array}$ & $\begin{aligned} r_{2}{ }^{*} & =s_{1}\left(1-r_{1}\right) \\
r_{5}{ }^{*} & =s_{2}\left(1-r_{4}\right) \\
r_{8}{ }^{*} & =s_{3}\left(1-r_{7}\right) \\
r_{11}{ }^{*} & =s_{4}\left(1-r_{10}\right) \\
r_{14}{ }^{*} & =s_{5}\left(1-r_{13}\right)\end{aligned}$ & $\begin{array}{c}r_{3}{ }^{*}=1-\left[1-s_{4}\left(1-c_{3}\right)\right]\left[1-s_{5}\left(1-c_{5}\right)\right] \\
r_{6}{ }^{*}=1-\left[1-s_{6}\left(1-c_{7}\right)\right]\left[1-s_{7}\left(1-c_{9}\right)\right] \\
r_{9}{ }^{*}=1-\left[1-s_{8}\left(1-c_{11}\right)\right]\left[1-s_{9}\left(1-c_{13}\right)\right] \\
r_{12}{ }^{*}=1-\left[1-s_{2}\left(1-c_{4}\right)\right] \\
r_{15}{ }^{*}=1-\left[1-s_{3}\left(1-c_{6}\right)\right] \\
r_{17}{ }^{*}=s_{6}\left(1-r_{16}\right) \\
r_{19}{ }^{*}=s_{7}\left(1-r_{18}\right) \\
r_{21}{ }^{*}=s_{8}\left(1-r_{20}\right) \\
r_{23}{ }^{*}=s_{9}\left(1-r_{22}\right)\end{array}$ \\
\hline
\end{tabular}

TABLE 2: Breakers anticipant estates.

\begin{tabular}{|c|c|}
\hline 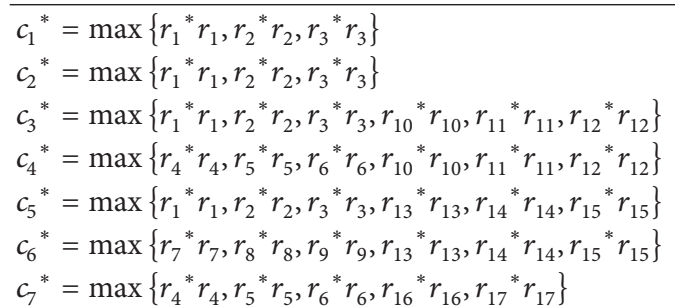 & $\begin{array}{l}{c_{8}}^{*}=\max \left\{r_{16}{ }^{*} r_{16}, r_{17}{ }^{*} r_{17}\right\} \\
{c_{9}}^{*}=\max \left\{r_{4}{ }^{*} r_{4}, r_{5}{ }^{*} r_{5}, r_{6}{ }^{*} r_{6}, r_{18}{ }^{*} r_{18}, r_{19}{ }^{*} r_{19}\right\} \\
c_{10}{ }^{*}=\max \left\{r_{18}{ }^{*} r_{18}, r_{19}{ }^{*} r_{19}\right\} \\
c_{11}{ }^{*}=\max \left\{r_{7}{ }^{*} r_{7}, r_{8}{ }^{*} r_{8}, r_{9}{ }^{*} r_{9}, r_{20}{ }^{*} r_{20}, r_{21}{ }^{*} r_{21}\right\} \\
{c_{12}}^{*}=\max \left\{r_{20}{ }^{*} r_{20}, r_{21}{ }^{*} r_{21}\right\} \\
c_{13}{ }^{*}=\max \left\{r_{7}{ }^{*} r_{7}, r_{8}{ }^{*} r_{8}, r_{9}{ }^{*} r_{9}, r_{22}{ }^{*} r_{22}, r_{23}{ }^{*} r_{23}\right\} \\
c_{14}{ }^{*}=\max \left\{r_{22}{ }^{*} r_{22}, r_{23}{ }^{*} r_{23}\right\}\end{array}$ \\
\hline
\end{tabular}

Corresponding elements estate vector is $S=\left[s_{1}, s_{2}, \ldots s_{9}\right]$, the breakers real estate vector is

$$
\begin{aligned}
C & =\left[c_{1}, c_{2}, c_{3}, c_{4}, c_{5}, c_{6}, c_{7}, c_{8}, c_{9}, c_{10}, c_{11}, c_{12}, c_{13}, c_{14}\right] \\
& =[1,1,0,1,1,1,0,0,0,0,1,0,0,0] .
\end{aligned}
$$

Corresponding breakers are CB1, CB3, CB4, CB5, CB6, CB7, CB9, CB10, CB11, CB12, CB13, CB14, CB15, and CB16. The protections real estate vector is

$$
\begin{aligned}
R & =\left[r_{1}, r_{2}, \ldots, r_{23}\right] \\
& =[0,0,1,0,0,0,0,0,0,0,1,0,1,0,0,0,0,0,0,0,1,0,0] .
\end{aligned}
$$

Corresponding protections are $\mathrm{B} 1 \mathrm{~m}, \mathrm{~B} 1 \mathrm{p}, \mathrm{B} 1 \mathrm{~s}, \mathrm{~B} 3 \mathrm{~m}, \mathrm{~B} 3 \mathrm{p}$, B3s, B4m, B4p, B4s, T1m, T1p, T1s, T2m, T2p, T2s, L3m, L3p, L4m, L4p, L5m, L5p, L6m, and L6p. The protections and breakers anticipant estates are shown in Tables 1 and 2 .

According to Formula (5), we can get the example's aim function, shown as Formula (8):

$$
\begin{aligned}
E(S)= & 10+\left(2 s_{1}+4\right)\left(1-s_{4}\right)+2 s_{2}+2 s_{3}-s_{4}-s_{5} \\
& +2 s_{6}+3 s_{7}-s_{8}+3 s_{9}-\max \left\{s_{4}, s_{5}\right\} .
\end{aligned}
$$

By means of calculation and communication among multiagents, we can get the result of this fault diagnosis model as follows: the failure elements are transformers T1 and T2 and line L5. From the results and analysis, we know that the main protection of transformer T1 refuses to act; breaker CB4 refuses to act; the main protection of line L5 refuses to act; the information of CB14 is missing.

\section{Conclusions}

Considering of the characteristics of agent, multiagent technique is suitable to be applied in fault diagnosis system for ship power system. Each agent can independently complete some simple tasks and accomplish complex tasks by communicating with other agents. Agents in the fault diagnosis system have clear division of functions; they can cooperate effectively to solve fault diagnosis problem for ship power system. The multiagent fault diagnosis system has both autonomy and interactivity; it is a new effective way for ship power system fault diagnosis.

\section{Conflict of Interests}

The authors declare that there is no conflict of interests regarding the publication of this paper.

\section{Acknowledgment}

This work is supported by National Natural Science Foundation of China under Grant no. 51177168.

\section{References}

[1] S. D. J. McArthur, E. M. Davidson, J. A. Hossack et al., "Automating power system fault diagnosis through multi-agent system," in Proceedings of the 37th Annual Hawaii International Conference on System Sciences, pp. 947-954, Big Island, Hawaii, USA, 2004.

[2] Z. Haiyan and X. Fei, "A survey of marine power system fault diagnosis," Ship Science and Technology, vol. 32, no. 4, pp. 134$137,2010$.

[3] J. Chen, J.-H. Wang, B. Zhang, and Z.-Y. Zhu, "Data fusion algorithm for ship power station fault diagnosis system," Electric Power Automation Equipment, vol. 26, no. 3, pp. 28-30, 2006.

[4] G. Cardoso, J. G. Rolim, and H. H. Zurn, "Application of neural-network modules to electric power system fault section 
estimation," IEEE Transactions on Power Delivery, vol. 19, no. 3, pp. 1034-1041, 2004.

[5] C. Liu, Z.-Y. He, and J.-W. Yang, "A quantum neural network based fault diagnosis algorithm for power grid," Power System Technology, vol. 32, no. 9, pp. 57-60, 2008.

[6] Y. Xu, Z. Lu, and Z. Guo, "Layered information fault diagnosis based on genetic algorithm," Power System Protection and Control, vol. 28, no. 10, pp. 15-18, 2000.

[7] J. Sun, S. Qin, and Y. Song, "Fault diagnosis method for power systems based on petri nets and probability information," Automation of Electric Power Systems, vol. 27, no. 13, pp. 10-14, 2003.

[8] Z. Zhang, R. Yuan, T. Yang, and M. Liu, "Rule extraction for power system fault diagnosis based on the combination of rough sets and niche genetic algorithm," Transactions of China Electrotechnical Society, vol. 24, no. 1, pp. 158-163, 2009.

[9] Y. Sun and Z. Liao, "Assessment of data mining model based on the different combination rough set with neural network for fault section diagnosis of distribution networks," Automation of Electric Power Systems, vol. 27, no. 6, pp. 31-35, 2003.

[10] H. Zhang, H. C. Yan, F. W. Yang, and Q. J. Chen, "Quantized control design for impulsive fuzzy networked systems," IEEE Transactions on Fuzzy Systems, vol. 19, no. 6, pp. 1153-1162, 2011.

[11] H. C. Yan, Z. Z. Su, H. Zhang, and F. W. Yang, "Observerbased $H_{\infty}$ control for discrete-time stochastic systems with quantization and random communication delays," IET Control Theory and Applications, vol. 7, no. 3, pp. 372-379, 2013.

[12] H. Zhang, H. C. Yan, F. W. Yang, and Q. J. Chen, "Distributed average filtering for sensor networks with sensor saturation," IET Control Theory and Applications, vol. 7, no. 6, pp. 887-893, 2013.

[13] H. C. Yan, H. B. Shi, H. Zhang, and F. W. Yang, "Quantized $H_{\infty}$ control for networked delayed systems with communication constraints," Asian Journal of Control, vol. 15, no. 5, pp. 14681476, 2013.

[14] H. J. Gao, T. W. Chen, and J. Lam, "A new delay system approach to network-based control," Automatica, vol. 44, no. 1, pp. 39-52, 2008.

[15] H. Zhang, Q. J. Chen, H. C. Yan, and J. H. Liu, "Robust $H_{\infty}$ filtering for switched stochastic system with missing measurements," IEEE Transactions on Signal Processing, vol. 57, no. 9, pp. 3466-3474, 2009.

[16] Z. Wang, L. Xia, and Y. Wang, "Application of multi-agent and genetic algorithm in network reconfiguration of ship power system," Elektronika ir Elektrotechnika, vol. 18, no. 9, pp. 7-10, 2012.

[17] Z. Wang, D. Zhao, Y. Wang, and D. Liu, "Reconfiguration of shipboard power system using discrete particle swarm optimisation," International Journal of Modelling, Identification and Control, vol. 15, no. 4, pp. 277-283, 2012.

[18] T. Nagata and H. Sasaki, "A multi-agent approach to power system restoration," IEEE Transactions on Power Systems, vol. 17, no. 2, pp. 457-462, 2002.

[19] H. Zhan and Q. Yan, "Application of multi-agent technology in power system," Journal of Chongqing University (Natural Science Edition), vol. 29, no. 11, pp. 53-57, 2006.

[20] H.-C. Shu, L. Tang, and J. Dong, "A survey on application of multi-agent system in power system," Power System Technology, vol. 29, no. 6, pp. 27-31, 2005.

[21] Y. Fan and J. Cao, Multi-Agent System Theory, Methods and Applications, Springer, Berlin, Germany, 2005.
[22] R. Khosla and Q. Li, "Multi-layered multi-agent architecture with fuzzy application in electrical power systems," in Proceedings of the IEEE International Conference on Fuzzy Systems, vol. 1, pp. 209-214, Honolulu, Hawaii, USA, May 2002.

[23] H. Zhang, H. C. Yan, T. Liu, and Q. J. Chen, "Fuzzy controller design for nonlinear impulsive fuzzy systems with time delay," IEEE Transactions on Fuzzy Systems, vol. 19, no. 5, pp. 844-856, 2011.

[24] H. Zhang, Z. H. Guan, and G. Feng, "Reliable dissipative control for stochastic impulsive systems," Automatica, vol. 44, no. 4, pp. 1004-1010, 2008.

[25] L. X. Zhang, H. J. Gao, and O. Kaynak, "Network-induced constraints in networked control systems-a survey," IEEE Transactions on Industrial Informatics, vol. 9, no. 1, pp. 403-416, 2013.

[26] H. Weng, P. Mao, and X. Lin, "An improved model for optimizing power system fault diagnosis," Automation of Electric Power Systems, vol. 31, no. 7, pp. 66-70, 2007. 


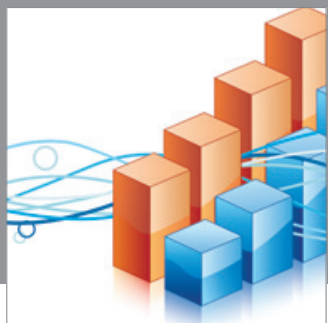

Advances in

Operations Research

mansans

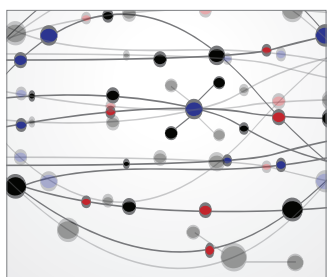

The Scientific World Journal
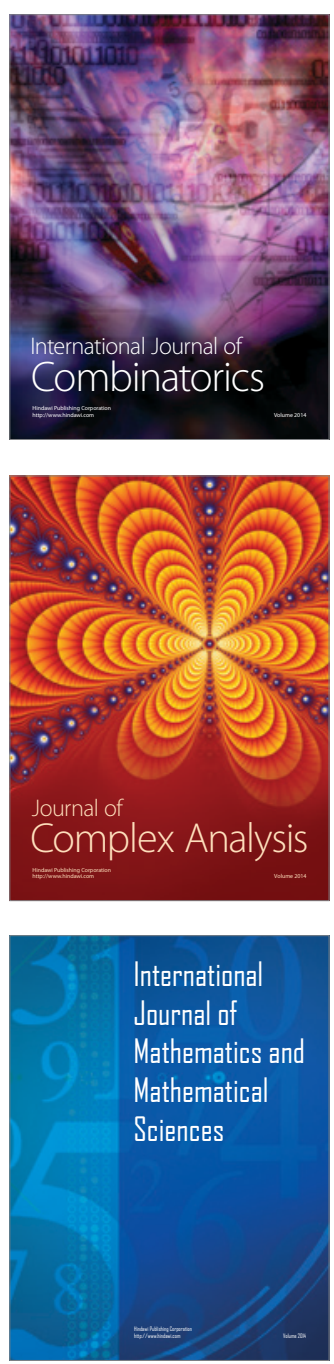
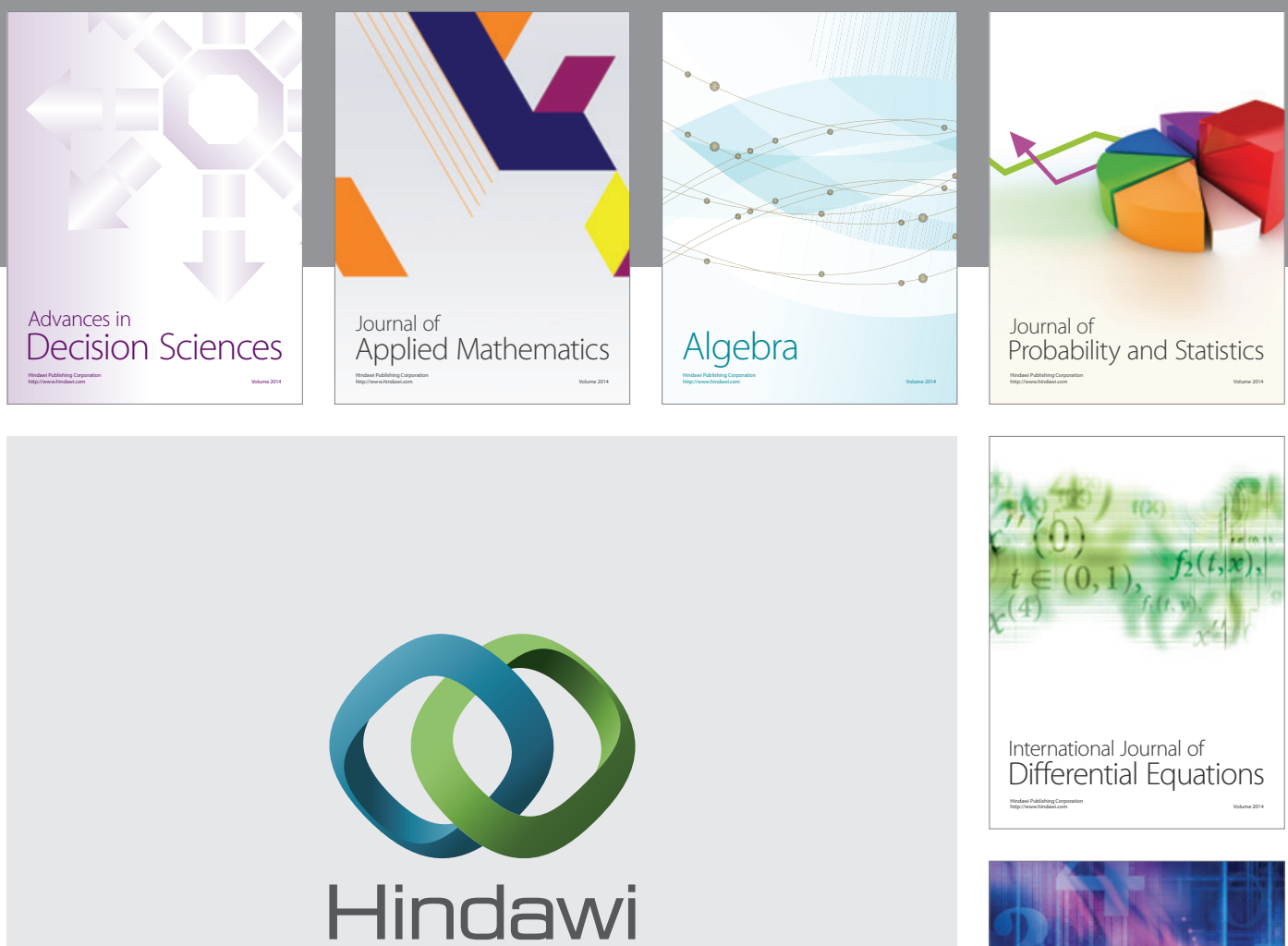

Submit your manuscripts at http://www.hindawi.com
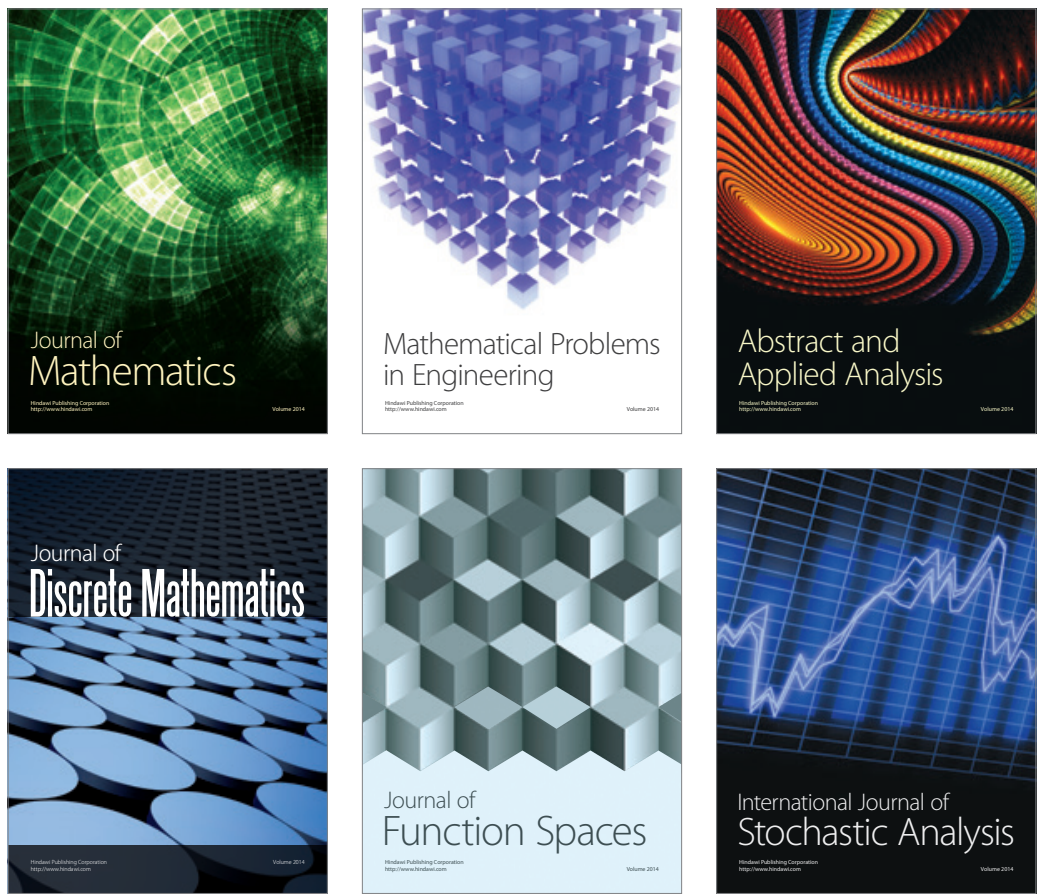

Journal of

Function Spaces

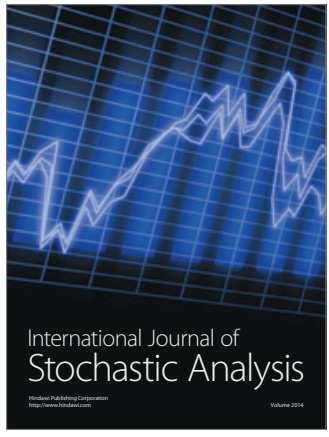

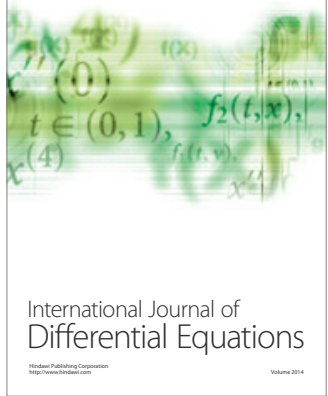
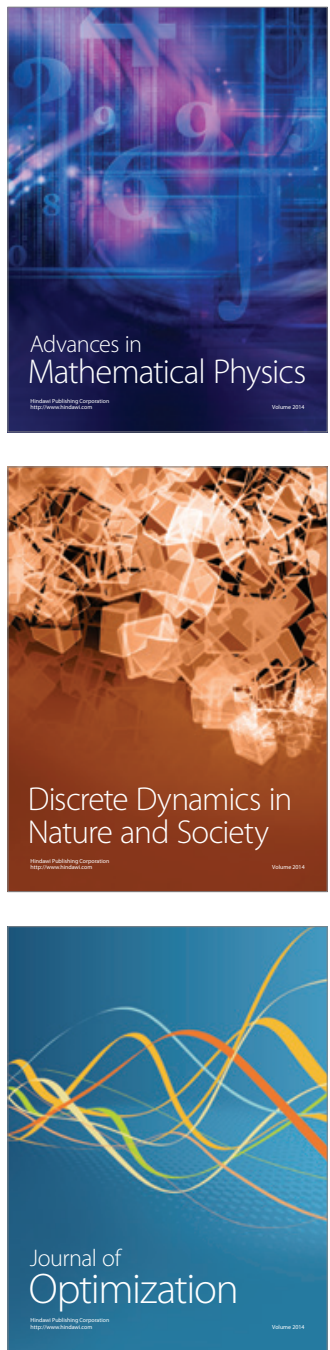